EDITORIAL

\title{
Risk stratification in acute coronary syndrome: focus on unstable angina/non-ST segment elevation myocardial infarction
}

\section{R Bugiardini}

Heart 2004;90:729-731. doi: 10.1136/hrt.2004.034546

\begin{abstract}
Although there have been advances in the management of unstable angina/non-ST segment elevation myocardial infarction syndromes, the rate of cardiovascular mortality after discharge is still unacceptably high. With many therapeutic options available, the clinician is challenged to identify the safest and most effective treatment for long term survival of each individual patient
\end{abstract}

"Simple, but not too simple" - Albert Einstein

$\mathrm{T}$ welve million individuals in the USA and 143 million worldwide have coronary artery disease. Two million US patients are admitted annually to cardiac care units with acute coronary syndromes (ACS). The number of hospital admission for patients with unstable angina/non-ST segment elevation myocardial infarction (UN/NSTEMI) is greater than the number with ST elevation myocardial infarction: 600000 and 1.4 million, respectively.

Extensive clinical trial data have provided substantial evidence to develop guidelines for risk stratification of UA/NSTEMI. ${ }^{3}$ However, short term mortality in clinical trials is approximately $2 \%$ compared with $4-5 \%$ mortality in clinical practice. ${ }^{4}$ The gap between today's knowledge in terms of volume of studies and guidelines and their application may provide only a partial explanation for the difference. Guidelines are prepared for physicians practising in primary and secondary centres, in most of which an invasive therapy is available. Guidelines are based on results of clinical trials. Patient enrolment in clinical trials is extremely selective. There are few women, patients are relatively young, and few patients have diabetes, heart failure or prior revascularisation. The overall consequence is that clinical trial guidelines could not mimic actual clinical practice. We need systems to minimise errors, to provide simple clinical models and scores to predict inhospital risk, comparative studies to support choices between alternative strategies, and evaluation of the impact of prognostic indexes on patient long term outcome.

\section{SOURCES OF ERROR}

Although applying guidelines is highly rational, clinicians do not often make decisions in this fashion. Experts in a variety of fields make decisions using a more intuitive process of recognising patterns and applying their own rules. In varying proportions, pathophysiologic reasoning, personal clinical experience, and recent published research each play a role in the development of our own clinical rules. This approach may produce incorrect use of tools of risk stratifications and inappropriate use of treatment strategies and procedures. However, errors are more often due to "failure" of the system, not of the doctors. Most errors occur at the transfer of care, and particularly at the transfer from the outpatient to the inpatient sites. There are a number of programs now focusing on errors and strategies to reduce errors (GAP, CRUSADE QI, JACHO). ${ }^{5-7}$ All of these programs focus on education of physicians, better interaction between health care organisations and physicians, and appropriate use of care pathways.

\section{SCORES TO PREDICT IN-HOSPITAL RISK}

Scores represent a simple, convenient method of risk stratification, in which a number of independent risk factors on presentation are shown to have prognostic significance. Independent risk factors may not necessarily represent "independent" pathophysiologic processes. Regression modelling techniques for prognostic analysis can be enhanced by the use of indexes that combine several clinical variables measuring different aspects of the same underlying pathophysiologic phenomenon. For example, the presence of high troponin $\mathrm{T}$ concentrations, congestive heart failure, low left ventricular ejection fraction, history of myocardial infarction, and Q waves on ECG all measure different aspects of the extent of myocardial damage. Placing each of these variables separately in a stepwise regression analysis might overlook the full importance of myocardial damage as a prognostic factor. A clinical index that combines the information provided from several related variables is a more powerful prognostic factor than any individual variable.

\footnotetext{
Abbreviations: ACS, acute coronary syndromes; GRACE, global registry of acute coronary events; PURSUIT, platelet glycoprotein Ilb/Illa in unstable angina: receptor suppression using Integrilin therapy; TIMI, thrombolysis in myocardial infarction; UA/STEMI, unstable angina/non-ST- segment elevation myocardial infarction
} 
The three major determinants of prognosis in ACS include: (1) the extent of myocardial injury; (2) the extent of coronary artery disease; and (3) the instability of the disease and its refractoriness to management. The GRACE and PURSUIT scores are prominent examples of scoring systems that give an overview of myocardial injury. ${ }^{8}$ These scores provided an excellent ability to assess risk for in-hospital and short term mortality. Age, Killip class, heart rate, systolic blood pressure, ST segment deviation, resuscitation from cardiac arrest, serum creatinine concentration, and raised cardiac enzymes were powerful predictors of prognosis in almost all patients.

The TIMI risk score seems to look more at the extent of coronary artery disease and its instability. ${ }^{10}$ Seven independent predictor variables were identified: age $>65$ years, three cardiovascular risk factors, known coronary artery disease (50\% stenosis), severe anginal symptoms (two episodes in preceding 24 hours), use of aspirin in the last seven days, ST segment deviation $>0.05 \mathrm{mV}$, and elevated serum cardiac markers of necrosis.

The advantage of these scoring systems is that they summarise important prognostic information of the disease in a single number, confidence limits can be easily calculated, and survival rates can be easily compared between given treatments.

\section{PROGNOSTIC INDEXES OF LONG TERM OUTCOME}

A major issue in the management of ACS is the risk stratification of those individuals who have survived the initial hospital admission without acute myocardial infarction, and are being prepared for discharge. Despite a substantial improvement in intra-hospital assessment and care, patients still have a considerably high incidence of medium to long term adverse outcomes. The risk continues to increase for at least 3-4 years after an episode of unstable syndromes. ${ }^{11}$ Death may range from $1.7 \%$ after one month to $9.5 \%$ after two years. Current areas of research try to address this issue. ${ }^{11-13} \mathrm{C}$ reactive protein, troponin $\mathrm{T}$, heart rate, ST segment depression on standard ECG, diabetes, congestive heart failure, creatinine clearance, $\mathrm{N}$-terminal pro-brain natriuretic peptide, old age, and use of two or more antianginal drugs at admission are independent long term predictors of death from cardiac causes. C reactive protein, $\mathrm{N}$ terminal pro-brain natriuretic peptide, and troponin $\mathrm{T}$ appear to be the markers with the strongest relation. These data are rational. Troponin is the most sensitive and specific marker of myocyte necrosis. C reactive protein is a marker of inflammation that contributes to atherosclerosis and its complications. Brain natriuretic peptide may reflect the underlying impairment in left ventricular function.

Other old and new prospective cohort studies have identified that ST shifts at 24 hour ECG recordings and autonomic imbalance are factors independently predictive of post-discharge outcome. ${ }^{14}{ }^{15}$ Long term risk stratification depends critically on the underlying mechanism of disease. A better understanding of the pathophysiologic significance of each prognostic factor is needed to quantify its strength and assess its relative importance in different subsets of patients.

\section{ESTIMATION OF LONG TERM PROGNOSIS}

Many studies have used survival probability as one of the simplest descriptors of prognosis. This statistic represents the probability that a patient will survive longer than a specified period of time. At one year follow up, patients who died at 10 days and 11 months discharge are considered equivalently. This approach has some limitations. For example, the strength of a prognostic factor may be greater during one period of time than another, which may underlie different pathogenetic substrates and obscure the perception of the best therapeutic strategy. In this issue of Heart, Nørgaard and colleagues ${ }^{16}$ provide further information in this area. They followed patients admitted with diagnosis of UA/NSTEMI for a median of 28 months. Independent predictors of cardiac death and acute myocardial infarction were age $>65$ years, previous myocardial infarction, congestive heart failure, ST segment shifts at 24 hour ECG recording, and troponin T. They estimated the probability of a patient being alive at any point in time after study entry. The prognostic value of transient ST segment shifts was confined to the medium term outcome (1-4 months). Troponin $\mathrm{T}$ provided long term prognostic information above and beyond conventional clinical risk markers such as previous myocardial infarction and congestive heart failure.

Data imply plausible pathogenetic mechanisms that fit with the widely accepted notion of the disease. ST segment shifts could reflect instability where persistence of acute stimuli may cause waxing and waning of intracoronary thrombosis and related transient myocardial ischaemia. High troponin values could correlate with both myocardial injury and extent of coronary disease. ${ }^{17}$

Effective therapeutic options for ACS are costly. We need studies to support choices. There is no consensus on how the long term use of many drugs should be undertaken. The link between ST segment shifts and medium term outcome could give insights into this matter. Detection of unstable patients as well as of a specific vulnerable period of time might be a rationale for the use of new, costly, potent antiplatelet and anti-inflammatory drugs in this setting.

\section{CONCLUSIONS}

Substantial advances in the management of UA/NSTEMI syndromes have occurred in the last decade. A disturbing note, however, is the recognition that the rate of cardiovascular mortality after discharge is still unacceptably high. With many therapeutic options available, the clinician is challenged to identify the safest and most effective treatment for long term survival of each individual patient. Steps in this process need simple prognostic markers that reflect pathophysiologic mechanisms to be identified. Empiric testing remains essential. Small cohort studies, clinical randomised trials, observational databases, and registries are all required to address this issue. Many innovations began with the report of few cases. The power of a key observation may be sufficient to demonstrate the potential of a new approach.

\section{REFERENCES}

1 National Center for Health Statistics. Detailed diagnoses and procedures: national hospital discharge survey, 1996. Hyattsville, Maryland: National Center for Health Statistics, 1998:13. Data from Vital and Health Statistics.

2 Braunwald E, Antman EM, Beasley JW, et al. ACC/AHA guideline update for the management of patients with unstable angina and non-ST-segment elevation myocardial infarction-2002: summary article: a report of the American College of Cardiology/American Heart Association task force on practice guidelines (committee on the management of patients with unstable angina). Circulation 2002;106:1893-900

3 Bertrand ME, Simoons ML, Fox KAA, et al. Management of acute coronary syndromes in patients presenting without persistent ST-segment elevation. Task force of the European Society of Cardiology. Eur Heart J 2002;23:1809-40.

4 Hasdai D, Behar S, Wallentin L, et al. A prospective survey of the characteristics, treatments and outcomes of patients with acute coronary syndromes in Europe and the Mediterranean basin; the Euro Heart Survey of acute coronary syndromes (Euro Heart Survey ACS). Eur Heart J 2002;23:1190-201.

5 Montoye CK, Mehta RH, Baker PL, et al. GAP steering committee. A rapidcycle collaborative model to promote guidelines for acute myocardial infarction. Jt Comm J Qual Saf 2003;29:468-78

6 Hoekstra JW, Pollack CV Jr, Roe MT, et al. Improving the care of patients with non-ST-elevation acute coronary syndromes in the emergency department: the CRUSADE initiative. Acad Emerg Med 2002;9:1146-55.

7 Keepnews D, Mitchell PH. Health systems' accountability for patient safety. Online J Issues Nurs 2003:8:2.

8 Granger CB, Goldberg RJ, Dabbous $O$, et al. Global registry of acute coronary events investigators. Predictors of hospital mortality in the global registry of acute coronary events. Arch Intern Med 2003;163:2345-53. 
9 PURSUIT Trial Investigators. Inhibition of platelet glycoprotein Ilb/Illa with eptifibatide in patients with acute coronary syndromes. Platelet glycoprotein Ill/Illa in unstable angina: receptor suppression using Integrilin therapy. N Engl J Med 1998;339:436-43.

10 Antman EM, Cohen M, Bernink PJLM, et al. The TIMI risk score for unstable angina/non-ST elevation $\mathrm{Ml}$ : a method for prognostication and therapeutic decision making. JAMA 2000;284:835-42.

11 Lindahl B, Toss H, Siegbahn A, et al. Markers of myocardial damage and inflammation in relation to long-term mortality in unstable coronary artery disease. FRISC study group. Fragmin during instability in coronary artery disease. N Engl J Med 2000;343:1139-47.

12 James SK, Lindahl B, Siegbahn A, et al. N-terminal pro-brain natriuretic peptide and other risk markers for the separate prediction of mortality and subsequent myocardial infarction in patients with unstable coronary artery disease. A global utilization of strategies to open occluded arteries (GUSTO)IV substudy. Circulation 2003;108:275-81.
13 Sabatine MS, Morrow DA, de Lemos JA, et al. Multimarker approach to risk stratification in non-ST elevation acute coronary syndromes. Simultaneous assessment of troponin I, C-reactive protein, and B-type natriuretic peptide. Circulation 2002;105:1760-3.

14 Manfrini O, Pizzi C, Trere D, et al. Parasympathetic failure and risk of subsequent coronary events in unstable angina and non-ST-segment elevation myocardial infarction. Eur Heart J 2003;24:1560-6.

15 Kennon S, Price CP, Mills PG, et al. Cumulative risk assessment in unstable angina: clinical, electrocardiographic, autonomic, and biochemical markers. Heart 2003:89:36-41.

16 Nørgaard BL, Andersen K, Thygesen K, et al. Long term risk stratification of patients with acute coronary syndromes: characteristics of troponin T testing and continuous ST segment monitoring. Heart 2004;90:739-44.

17 defilippi C, Wasserman S, Rosanio S, et al. Cardiac troponin T and C-reactive protein for predicting prognosis, coronary atherosclerosis, and cardiomyopathy in patients undergoing long-term hemodialysis. JAMA 2003;290:353-9.

\section{IMAGES IN CARDIOLOGY}

\section{Percutaneous device closure of post-infarction ventricular septal defect with aneurysm}

$\mathrm{V}$ entricular septal defect in association with left ventricular aneurysm formation has been reported rarely. Quinn and Brady (Heart 2002;87:567) describe attempted surgical closure of such a defect that was unsuccessful. A 61 year old man presented with a very similar defect 10 days after inferoseptal myocardial infarction. He was referred to our centre for consideration of surgical closure but deteriorated further with ventricular dysrhythmia, renal impairment, and hepatic congestion (AST 2078 units). An intra-aortic balloon pump was inserted with clinical improvement and surgery was considered. Transthoracic echocardiography demonstrated a large defect in the basal part of the muscular ventricular septum with an aneurysm bulging into the right ventricle. The aneurysmal portion was perforated (panel A, arrow) with colour flow Doppler studies demonstrating a significant left to right shunt (panel B). Coronary angiograms showed occlusion of only the left ventricular branch of the right coronary artery.

There was concern about a surgical approach through the left ventricle partly because the territory of either the patent left anterior descending artery or the patent posterior descending artery would be sacrificed and partly in view of the recent unfavourable report in Heart.

A percutaneous approach was therefore undertaken. The defect in the ventricular septum itself was too large to accept a device. After balloon sizing via a complete arterio-venous loop
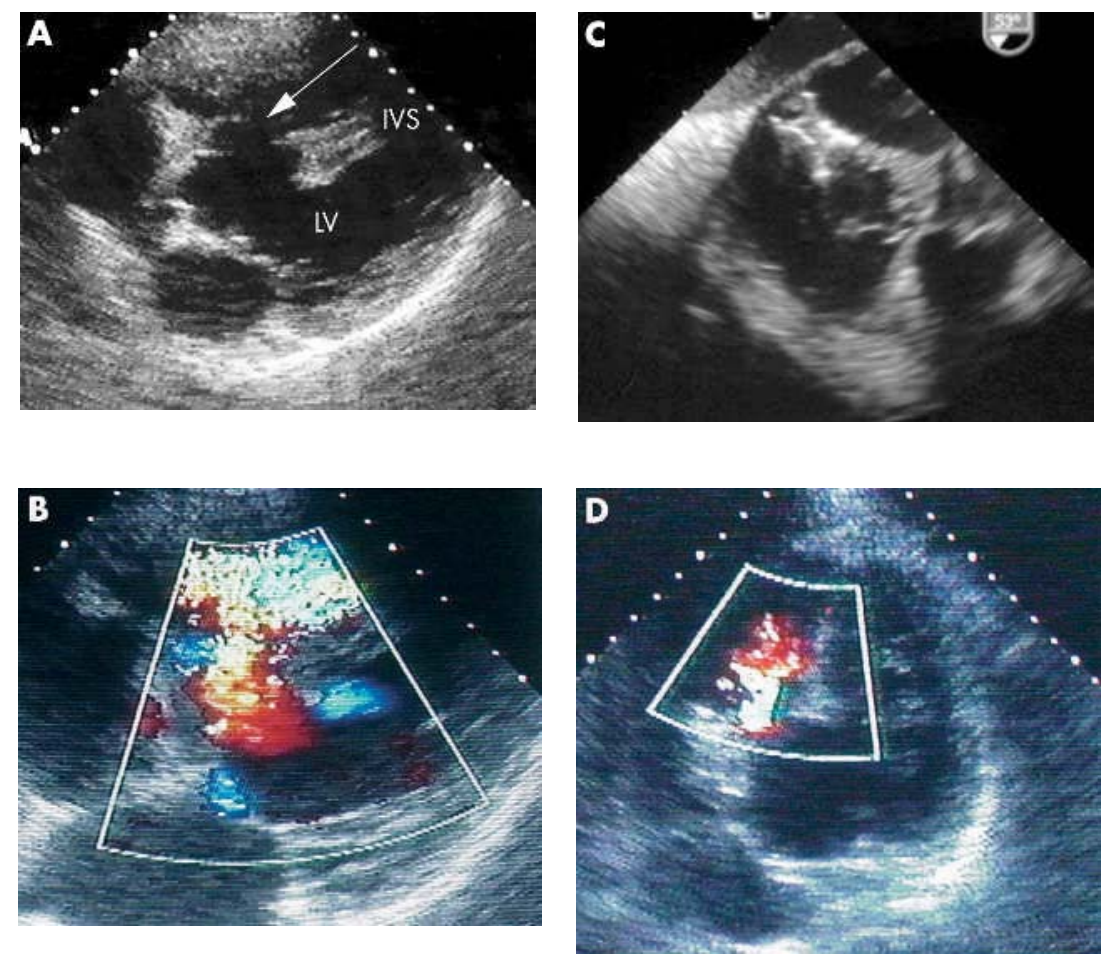

(from the right femoral artery across the defect to the right internal jugular vein), an $18 \mathrm{~mm}$ Amplatzer muscular ventricular septal defect closure device (AGA Medical Corporation, USA) was deployed into the largest perforation in the aneurysmal portion (panel C).

The aortic systolic pressure immediately increased by approximately $20 \mathrm{~mm} \mathrm{Hg}$, the intra-aortic balloon pump was removed at 24 hours, and the patient was discharged home at seven days. At two months follow up, he remained well and was walking 2-3 miles per day. Repeat transthoracic echocardiography demonstrated no flow across the device, but there was a residual shunt adjacent to it (panel D), which may require a second device.

C J Burrell

L A Zacharkiw

J V deGiovanni

frances.roach@phnt.swest.nhs.uk 